\title{
Knowledge and Practice on Oral Care among the Patients Receiving Chemotherapy
}

\author{
Acharya Radha ${ }^{1, *}$, Ojha Namrata ${ }^{2}$ \\ ${ }^{1}$ Kathmandu University School of Medical Sciences, Dhulikhel, Kavre \\ ${ }^{2}$ Emergency Department, Shahid Gangalal National Heart Centre, Kathmandu \\ *Corresponding author: radhapnd@yahoo.com
}

Received May 01, 2013; Revised November 11, 2013; Accepted February 08, 2014

\begin{abstract}
Changes to the oral cavity arising from malignant disease, treatment and other co-morbidity factors can have a profound affect on the person with cancer, causing pain, discomfort, lack of nutrition, longer hospital stays, and in some situations sepsis and death. While it is recognized that caring for the mouth is an important aspect of cancer care, all too often this aspect of care may be overlooked until problems arise. This leads to needless distress and discomfort and in some cases serious clinical consequences. Oral and gastrointestinal mucositis can affect up to $100 \%$ of patients undergoing high-dose chemotherapy and hematopoietic stem cell transplantation. The aim of the study was to assess knowledge and practice on oral care among the patients receiving chemotherapy. This is descriptive cross-sectional study. A total of 102 respondents, visiting B.P. Koirala Memorial Cancer Hospital, Bharatpur Chitwan and who met eligible criteria were systematically sampled and interviewed face to face. Descriptive and inferential statistics were used for the analysis using the SPSS version 16.0. The duration of the study was one month i.e. June, 2010. The tool was developed after reviewing the related literatures. The knowledge on oral care was adequate among $23.4 \%$ of the respondents and $18.6 \%$ of the respondents had adequate practice receiving chemotherapy. Only $28.4 \%$ respondents had adequate knowledge. Among total respondents $60.7 \%$ of the respondents had adequate knowledge on the prevention of oral problems and $55.8 \%$ of the respondents had adequate knowledge on treatment of oral problems. Nurses were found as a main source of information on oral care. Thus, it was concluded that majority of the respondents had inadequate knowledge and practice on oral care. There was association between selected demographic (age and education). There was no association between family history of cancer and knowledge of respondents. Thus the study also concluded that adequate knowledge on oral care should be provided to the patients before undergoing chemotherapy.
\end{abstract}

Keywords: knowledge, practice, oral care

Cite This Article: Acharya Radha, and Ojha Namrata, "Knowledge and Practice on Oral Care among the Patients Receiving Chemotherapy.” American Journal of Cancer Prevention, vol. 2, no. 1 (2014): 9-13. doi: 10.12691/ajcp-2-1-4.

\section{Introduction}

Cancer is a second most frequent cause of death in developed countries after cardiovascular disease and epidemiological evidence points to this trend emerging in the less developed countries. Oncology management includes multiple treatments dealing with the preventive, curative and rehabilitative aspects of malignant disease. Chemotherapy is one of the best modalities of treatment. In chemotherapy systemic drugs are used which kill cancer cell and is used as curative or palliative treatment [1].

There is clear evidence that oral changes, including the development of mucositis can lead to many serious and distressing problems in the cancer setting. These may include severe pain and discomfort leading to an inability to eat, drink, swallow or talk affecting the person's ability to recover and their quality of life. The alteration of the normal oral mucosa flora and the breakdown of the oral lining may also lead to an increased risk of local and systemic infections [2]. These complications increase morbidity, sometimes requiring increased hospital stays, a need to decrease the dosage, delay or stop necessary cytotoxic treatment. All of this leads to increased physical, emotional and financial costs and in some cases may result in death.

Approximately 400000 cases have treatment induced damage to oral cavity. Oral mucositis induced by chemotherapy is frequent occurring toxicity in patients. Severe mucositis has a major impact on patients daily functioning, well being and quality of life. The incidence of oral problems is range from $15-40 \%$ in patients receiving chemotherapy and $70-90 \%$ in bone marrow recipients receiving chemotherapy [3].

Mucositis can simply be defined as inflammation of the gastrointestinal tract, however in the cancer setting it usually refers to the erythematic, inflammation and ulceration that occurs in the mucosal lining of the mouth, pharynx, esophagus, and the entire gastrointestinal tract secondary to cytotoxic (chemotherapy, radiation) 
treatment [4]. In Bellm et al's [5] study to gain a better understanding of 38 patients' experiences while undergoing Hematology Stem Cell Transplantation (HSCT), patients reported mouth sores, nausea and vomiting, and fatigue as the most troubling side effects of the treatment. Mouth sores were selected as the single most debilitating side effect (42\%), followed by nausea and vomiting (13\%). Many of the patients mentioned that mouth sores made it difficult or impossible to eat $(n=23)$, swallow $(n=21)$, drink $(n=17)$, and talk $(n=8)$. Twenty patients reported pain in the mouth, throat and/or esophagus. Two thirds of the patients reported receiving opioid analgesia to relieve oral pain.

Oral problems includes oral mucositis, oral thrush, loss of taste sensation, dry mouth, burning mouth syndrome, bleeding gums, eating swallowing difficulties, soreness or pain in the mouth, dental caries. Severe adverse reactions due to these therapies result in patient morbidity and mortality. In addition it contributes to economic ramifications [6].

\section{Methods}

Descriptive cross sectional research design was used in Day Care Unit of B.P. Koirala Memorial Cancer Hospital (BPKMCH). It is the largest cancer hospital of Nepal. Study population consisted of all patients who have received at least one cycle of chemotherapy attending $\mathrm{BPKMCH}$ and who were present at the time of data collection. A total of 102 respondents, who met eligible criteria were systematically sampled and interviewed face to face.

A structured and semi-structured interview schedule consisting of questions related demographic characteristics and knowledge and practice developed by reviewing literature. The content validity of the instrument was established seeking opinion of oncologist and related experts nurses and doctors. The instrument was then translated into Nepali language and opinion of language expert was obtained for comprehensibility and simplicity of language and for consistency of the content. The study was conducted after obtaining approval from the concerned authority.

All patients who met the criteria and who gave informed consent were interviewed. Review of the patient's medical record file was done to confirm the number of cycle of chemotherapy.

The collected data were reviewed daily for completeness and accuracy. Edited data were entered into the Statistical Package for Social Science Software (SPSS) version 16.0 for statistical analysis using descriptive statistics.

Table 1. Knowledge on Types of Oral Problems

\begin{tabular}{|l|l|c|c|}
\hline S.N. & Types of oral problems & Frequency & Percentage (\%) \\
\hline 1. & Mucositis & 92 & 90.2 \\
\hline 2. & Dry mouth & 82 & 80.4 \\
\hline 3. & Taste changes & 58 & 56.9 \\
\hline 4. & Dental problems & 44 & 43.1 \\
\hline 5. & Oral pain & 23 & 22.5 \\
\hline 6. & Eating/swallowing difficulties & 17 & 16.7 \\
\hline 7. & Bleeding gums & 15 & 14.7 \\
\hline 8. & Blisters in tongue & 11 & 10.8 \\
\hline 9. & Gum swelling & 5 & 4.9 \\
\hline
\end{tabular}

\section{Results}

The above table shows that among 102 respondents, $90.2 \%$ (92) responded that mucositis is a type of oral problem. Similarly $80.4 \%$ (82) responded that dry mouth can also be caused as an oral side effect and 56.9\% (58) knew that taste change is also an oral problem. 43.1\% (44) of the respondents responded that dental problems are also oral problems. Minimum 4.9\% (5) respondents knew that gum swelling is also an oral problem caused by chemotherapy.

Table 2. Knowledge on Oral Problems $(n=102)$

\begin{tabular}{|c|c|c|c|}
\hline S.N. & Oral problems & Frequency & Percentage (\%) \\
\hline 1. & Mucositis is a commonly occurring oral problem & 49 & 48 \\
\hline 2. & Oral problems can lead to malnutrition & 39 & 38.2 \\
\hline 3. & Preventive measures should begin before the treatment & 18 & 17.6 \\
\hline 4. & Smoking and alcohol have adverse role in the prevention of oral problems & 96 & 94.1 \\
\hline 5. & Dental check up should be done at least two weeks before chemotherapy & 5 & 4.9 \\
\hline 6. & Soft brush can prevent oral problems & 89 & 87.3 \\
\hline 7. & Medicated mouth washes prevent oral problems & 102 & 100 \\
\hline 8. & Frequent sips of water should be taken for dry mouth & 88 & 86.3 \\
\hline
\end{tabular}

Table 2 illustrate that the knowledge on mucositis as a commonly occurring oral problem was among 48\% (49) of the respondents. The table also shows that $17.6 \%$ (18) of the respondents knew that preventive measures should begin before the treatment begins and $94.1 \%$ (96) of the population responded that smoking and alcohol have adverse role in the prevention of oral problems. Similarly very low percentage of population i.e. $4.9 \%$ (5) responded that dental checkup should be done at least two weeks before starting chemotherapy. Similarly, the majority of the population i.e. $87.3 \%$ (89) responded that use of soft brush can prevent oral problems while $100 \%$ (102) of the respondents responded that medicated mouth washes can be used to prevent oral problems. This signifies that majority of the respondents are aware of it. $86.3 \%$ (88) of the respondents responded that frequent sips of water should be taken for dry mouth.

The Table 3 shows that $30.4 \%$ (31) respondents responded that eating a well balanced diet can prevent oral problems and $87.3 \%$ (89) respondents knew that proper oral care can prevent oral problems. Similarly, $39.2 \%$ (40) respondents said that complete oral health exam and $16.7 \%$ (17) said that use of medication can prevent oral problems.

Table 3. Knowledge on Preventive Measures of Oral Problems $(\mathrm{n}=$ 102)

\begin{tabular}{|l|l|c|c|}
\hline S.N. & Preventive measures & Frequency & Percentage (\%) \\
\hline 1. & Proper oral care & 89 & 87.3 \\
\hline 2. & Complete oral health exam & 40 & 39.2 \\
\hline 3. & Eating a well balanced diet & 31 & 30.4 \\
\hline 4. & Use of medications & 17 & 16.7 \\
\hline
\end{tabular}


Table 4. Knowledge on Treatment of Oral Problems $(n=102)$

\begin{tabular}{|l|l|c|c|}
\hline S.N. & Treatment of oral problems & Frequency & Percentage (\%) \\
\hline 1. & Use of medicines & 79 & 77.5 \\
\hline 2. & Proper oral care & 77 & 75.5 \\
\hline 3. & Proper dental checkup & 22 & 21.6 \\
\hline
\end{tabular}

Among 102 respondents, 75.5\% (77) respondents knew that proper oral care is necessary for its treatment while only $21.6 \%$ (22) respondents knew that proper dental check up is also necessary for its treatment. Similarly, majority of the respondents i.e. $77.5 \%$ (79) responded that use of medications can treat oral problems.

Table 5. Practice on Oral Care $(n=102)$

\begin{tabular}{|l|l|c|c|}
\hline S.N. & Oral care practice & Frequency & Percentage (\%) \\
\hline 1. & Use of tooth brush and tooth paste for cleaning teeth daily & 98 & 96.1 \\
\hline 2. & Use soft brush & 71 & 69.6 \\
\hline 3. & Brush teeth twice a day & 43 & 42.2 \\
\hline 6. & Brush teeth more than two minutes & 74 & 72.5 \\
\hline 7. & Clean mouth while brushing teeth & 92 & 90.2 \\
\hline 8. & Use medicated mouth washes for oral care & 63 & 61.8 \\
\hline 9. & Take soft diet & 18 & 17.6 \\
\hline 11. & Changes brush every monthly & 26 & 25.5 \\
\hline 12. & Right practice of brushing teeth & 25 & 24.5 \\
\hline 14. & Inspect of oral cavity once a day & 26 & 25.5 \\
\hline
\end{tabular}

The above table indicates that out of 102 respondents majority of the respondent's i.e. 96.1\% (98) used tooth paste and tooth brush for cleaning their teeth daily. The practice of using soft brush was only among 69.6\% (71) of the respondents. Forty two percent (43) of the respondents had practice of brushing teeth twice a day. Similarly, $72.5 \%$ (74) of the respondents had practice of brushing teeth more than two minutes. The majority of the respondents, i.e. $90.2 \%$ (92) respondents had practice of cleaning mouth while brushing teeth. Only 61.8\% (63) of the respondents had practice of using medicated mouth washes. Among total respondents, 25.5\% (26) respondents changed their tooth brush every month. Only $24.5 \%$ (25) had right practice of brushing teeth and $25.5 \%$ (26) of the respondents had practice of inspection of oral cavity once a day.

\section{Discussion}

Among total respondents, $28.4 \%$ of the respondents had adequate knowledge regarding types of oral problems with mean score value 5.94 and standard deviation 3.17. The findings were contrast with Hoad-Reddick and health's questionnaire which found that knowledge of problems related to dental health was adequate just over half (52.6\%).

Mucositis as an oral problem was responded by $90.2 \%$ (92) respondents and 56.9\% (58) respondents responded that taste alteration as an oral problem while $80.4 \%$ (82) respondents said that dry mouth is also an oral problem. Similarly, only $43.1 \%$ (44) respondents knew that dental problems as an oral problem whereas gum bleeding was responded by $14.7 \%$ (15) of the respondents.

Among total respondents $21.6 \%$ (22) of the respondents knew that tooth decay as a dental problem while only $14.7 \%$ (15) of the respondents responded that tooth pain can also be caused as a dental problem of chemotherapy. Dental plaque was responded by $10.8 \%$ (11) of the respondents and only $5.9 \%$ (6) of the respondents responded that loose teeth can also be caused as a side effect of chemotherapy.

In this study it was found that $60.7 \%$ of the respondents had adequate knowledge regarding prevention on oral problems with mean score value 6.03 and standard deviation 1.46. Among total respondents, 55.8\% respondents had knowledge regarding treatment of oral problems with mean score value 3.85 and standard deviation 1.26. The results were not similar with questionnaire survey of Roberts [6] which reported that the current knowledge about prevention and treatment of oral problems was $71 \%$.

Only $17.6 \%$ (18) of the respondents knew that the preventive measures should begin before the treatment starts. Majority of the respondents i.e. $75.5 \%$ (77) respondents responded that proper oral care is required for the treatment of oral problems while $87.3 \%$ (89) respondents responded that proper oral care is necessary for the prevention of oral problems.

Thirty percent (31) respondents responded that balanced diet is essential for the prevention of oral problems while only $39.2 \%$ (40) respondents knew that oral health exam is essential for its prevention. Only $16.7 \%$ (17) respondents responded that use of medications can prevent oral problems. Regarding treatment of oral problems $77.5 \%$ (79) of the respondents responded that medications can treat oral problems while only $21.6 \%$ (22) of the respondents knew that dental check up is necessary for the treatment of oral problems as a side effect of chemotherapy.

Among total respondents $38.2 \%$ (39) of the respondents said that oral problems can lead to malnutrition. Similarly, $86.3 \%$ (88) of the respondents knew that frequent sips of water should be taken for the management of dry mouth. $35.3 \%$ (36) respondents responded that dental check up is necessary for the prevention of oral problems but surprisingly none of the respondents had dental check up before starting chemotherapy. Similarly 87.3\% (89) respondents responded that use of soft brush can prevent oral problems and 100\% (102) respondents responded that use of medicated mouth washes can be used to prevent oral problems but the practice of using soft brush was seen in only $69.3 \%$ (71) of the respondents and use of medicated mouth washes was reported in only $61.8 \%$ (63) of the respondents.

In the research in Iran it was reported by Eilers [7], that $57.5 \%$ of the respondents used soft brush and $10 \%$ of the patients who were suffering from ulcer of mouth reported that they applied sodium bicarbonate as mouth wash solution which shows that the practice of using soft brush and medicated mouth washes was satisfactory but not consistent with the knowledge they possessed. 
The study revealed that only $11.8 \%$ (12) had heard about fluoride, the practice of taking soft diet was only among $17.6 \%$ (18) respondents and likewise the practice of daily inspection of oral cavity was only among $25.5 \%$ (26) respondents. None of the respondents had practice of using fluoride gel, flossing teeth and softening tooth brush in warm water. The findings were similar with a study on oral care in nursing which stated that self-rated knowledge was poorest on fluorides and daily examination of the oral cavity was performed by only $6 \%$ of the respondents which is inconsistent with this research finding [8].

Among 102 respondents 24.5\% (25) respondents had right practice of brushing teeth however, 29.4\% (30) respondents had faced oral problems during their treatment and among the 30 respondents 90\% (27) respondents answered that they focused on oral care after having oral problems. A study done by Stone \& Hood [9] in Korea, the right practices of the respondents regarding brushing teeth was $30 \%$ which is consistent with this study.

The overall adequate knowledge regarding oral care was found among $23.5 \%$ (24) of the respondents with mean score value 15.82 and standard deviation 5.08 while the adequate practice was found only among $18.6 \%$ (19) of the respondents with mean score value 5.07 and standard deviation 2.41. The knowledge and practice was found to be relatively low among the respondents which was not consistent with Stone and Hood's [9] questionnaire survey which found that whilst knowledge of problems related to dental health was adequate just over half $(52.6 \%)$. The findings were contrast with the research in Iran which revealed that overall correct responded answers were $30 \%$ in knowledge part and 53\% in practice part.

Overall, the knowledge and practice on oral care was inadequate and relatively lower compared to the previous research results which may be a result of inadequate knowledge regarding the side effects of chemotherapy among the health care providers or due to the communication gap between them and the patients.

In the study the knowledge is compared with the demographic variables such as age, education, family history of cancer. The association between information provided on oral care and knowledge of the respondents and the association between knowledge of oral care and practice on oral care was also seen. The findings revealed that there was association between age, education and knowledge of the respondents $(p<0.05)$. The findings were contrast with the study by Adams [1] which concluded that there was no significant relation of knowledge neither with age nor with education [11].

The study also concluded that there was association between information on oral care and knowledge on oral care $(\mathrm{p}<0.05)$. There was no association was seen between family history of cancer and knowledge on oral care. The finding was similar with the same research in Iran which showed that there was no significant relation between knowledge with family history of cancer [10].

The information on oral care was provided to maximum respondents though the knowledge and practice on oral care was inadequate among majority of the respondents which suggests lack of continuous monitoring and lack of awareness on oral health among the patients.
The studies have shown that the oral problems are high among the patients receiving chemotherapy. Oral problems in cancer patients cover a wide range. The mouth is frequent site of complication associated with chemotherapy. Forty percent rise in mucositis was noted in 1996-2000 compared with 1991-1995. Study has shown that $17-40 \%$ of the clients receiving chemotherapy get oral problems [4].

Cancer specialists state that approximately $40 \%$ of chemotherapy patients will have oral problems, but this number reduces to about $12 \%$ if their treatment is integrated beforehand. Improvement in physician/health worker and patient communication can result in better patient care and help patients adapt to illness and treatment and improve treatment approaches. Although extra care is focused in chemotherapy but due to inadequate knowledge and practice oral problems yet cannot be avoided [11].

The study also reveals that the percentage of patients' awareness about chemotherapy complication on oral problems was only $27.5 \%$. In the study $10 \%$ of patients were suffering from ulcers of mouth reported that they apply sodium bicarbonate solution as mouth wash solution and 57.5 used soft brush. In relation to knowledge, 30\% had correct answer, 33\% had wrong answer and 37\% answered "I don't know". Similarly in the practice part $53 \%$ had correct and $47 \%$ had wrong practices regarding prevention and management of drug complications. The study showed that there was no significant relation between both knowledge and practice of the women with marital status, living place and family history of cancer. The study also revealed that women who were attending to receive third episode had more knowledge than they were referring for the first episode [12].

In summary the study showed that the knowledge of patients directly affects their practice. To upgrade the practice proper knowledge should be provided to the patients from the very beginning of the therapy. Likewise dental examination is a key component to limit the oral problems and the practice of dental exam in our setting before starting chemotherapy is almost nil which suggest the ignorance in the preventive aspects of the care providers.

\section{Conclusion}

The knowledge on oral care was adequate among 24 of the respondents. Similarly, the knowledge regarding the types of oral problems was lowest i.e. only 29 respondents had adequate knowledge. Among 102 respondents 60.7\% of the respondents had adequate knowledge on the prevention of oral problems and 55.8\% of the respondents had adequate knowledge on treatment of oral problems.

The study revealed that 19 of the respondents had adequate practice. While among 30 respondents who faced oral problems $90 \%$ of the respondents responded that they focused on oral care. This shows that if the proper education was provided to the patients regarding the oral problems and oral care the knowledge and practice would both have been adequate.

There was association between the education, age, information regarding oral care and respondents' knowledge on oral care with $\mathrm{p}<0.05$. There was also 
association between knowledge regarding oral care and practice regarding oral care (not showing in table). Thus it concludes that information regarding oral care is essential to acquire adequate knowledge and practice on oral care whereas the knowledge doesn't differ whether there is family history of cancer or not.

\section{References}

[1] Adams, R. (1996). Qualified nurse's lack adequate knowledge related to oral health, resulting in adequate oral care of patients on medical wards, Journal of advanced, pp. 552-560.

[2] Sonis ST (2004). The Pathobiology of Mucositis. Nature Reviews Cancer. 4: 277-284. Stone R. \& Hood (2007). mangement of oral mucositis at European transplantation centres, European journal of oncology nursing, pp. 53-59.

[3] Cawley, M. \& Laura, M. (2005). Current Trends in Managing Oral Mucositis. Clinical Journal of Oncology Nursing, 9, (5): pp. 584592.

[4] Dodds, MJ. et al. (2004). The Pathogenesis and Characterisation of Oral Mucositis Associated with Cancer Therapy. Oncology Nursing Forum. 31 (4): 5-11.
[5] Bellm, LA, Epstein, JB, Rose-Ped, A, et al. (2000). Patient Reports of Complications of Bone Marrow Transplantation. Support Care Cancer. 8: 33-39.

[6] Roberts, J. (2005). Developing an oral assessment and intervention tool for older people. British Journal of Nursing, 9 (17), pp. 11241127, Vol 6, No 1, pp. 28-32.

[7] Eilers, J. (2004). Nursing interventions and supportive care for the prevention and treatment of oral mucositis associated with cancer treatment. Oncology Nursing Forum, 31, (4), pp. 13-23.

[8] Djuric, M (2006). Mucositis prevention by improved dental care in acute leukemia patients. Support Care Cancer, pp. 137-146.

[9] Stone J E \& Hood H V (2007). Interventions for preventing oral mucositis for patients with cancer receiving treatment (Cochrane Review). The Cochrane Library, Issue 4, Chichester, UK: John Wiley \& Sons.

[10] Andersson, P., Westergren, A., Karlsson, S., Hallberg I. R., \& Renvert, S. (2002). Oral health and nutritional status in a group of geriatric rehabilitation patients. Scandinavia Journal of Caring Science, Vol. 16, pp. 311-318.

[11] Rubenstein, E, \& Perterson, S. (2008). Clinical Practice Guidelines for the Prevention and Treatment of Cancer TherapyInduced Oral and Gastrointestinal Mucositis. Supplement to Cancer, 100, (9) pp. 2026-2033.

[12] Haghpanah, S. M., Aminni, M., Kherad K. (2006), knowledge and practice of patients with breast cancer patients about complication of chemotherapy, J Res Health Science, Vol 6, No 1, pp. 28-32. 